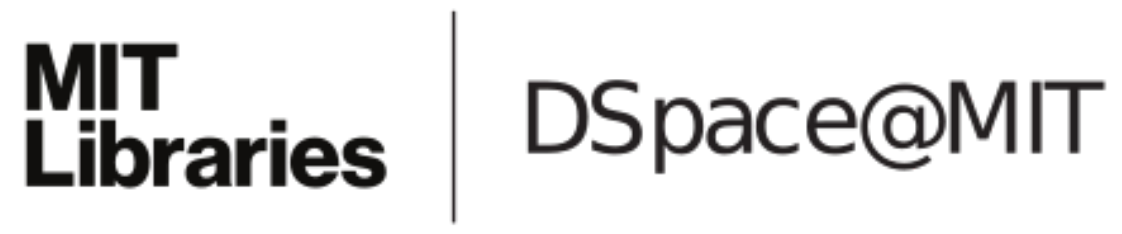

\author{
MIT Open Access Articles
}

An Adaptive Control Technology for Safety of a GTM-like Aircraft

The MIT Faculty has made this article openly available. Please share how this access benefits you. Your story matters.

Citation: Matsutani, M. et al. "An adaptive control technology for safety of a GTM-like aircraft." American Control Conference, 2009. ACC '09. 2009. 3238-3243. (C) 2010 IEEE.

As Published: http://dx.doi.org/10.1109/ACC.2009.5160747

Publisher: Institute of Electrical and Electronics Engineers

Persistent URL: http://hdl.handle.net/1721.1/60386

Version: Final published version: final published article, as it appeared in a journal, conference proceedings, or other formally published context

Terms of Use: Article is made available in accordance with the publisher's policy and may be subject to US copyright law. Please refer to the publisher's site for terms of use. 


\title{
An Adaptive Control Technology for Safety of a GTM-like Aircraft
}

\author{
Megumi Matsutani, Travis Gibson, Jinho Jang, Luis G. Crespo, and Anuradha Annaswamy
}

\begin{abstract}
An adaptive control architecture for safe performance of a transport aircraft subject to various adverse conditions is proposed and verified herein. This architecture combines a nominal controller based on an LQR with integral action, and an adaptive controller that accommodates for actuator saturation and bounded disturbances. The effectiveness of the baseline controller and its adaptive augmentation are evaluated and compared using a stand-alone control verification methodology. Several failure modes, where an uncertain parameter and a correspondingly critical flight maneuver are paired, are studied. The resilience of the controllers is determined by evaluating the degradation in closed-loop performance that results from increasingly larger uncertainties. Symmetric and asymmetric actuator failures, flight upsets, and CG movements, are some of the uncertainties considered.
\end{abstract}

\section{INTRODUCTION}

The challenge of achieving safe flight comes into sharp focus in the face of adverse conditions caused by faults, damage, or upsets. An appropriate technology that has the potential for enabling a safe flight under these adverse conditions is adaptive control. One of the main features of an adaptive control architecture is its ability to react to changing characteristics of the underlying aircraft dynamics.

The field of adaptive control is a mature theoretical discipline that has evolved over the past thirty years, embodying methodologies for controlling uncertain dynamic systems with parametric uncertainties [1], [2], [3]. Through the efforts of various researchers over this period, systematic methods for the control of linear and nonlinear dynamic systems with parametric and dynamic uncertainties, stability and robustness properties of these systems in the presence of disturbances, time-varying parameters, unmodeled dynamics, time-delays, and various nonlinearities, have been outlined in several journal and conference papers over the past three decades. What is needed therefore is a systematic deployment and evaluation of these methods for the problem of ensuring safe flight in an aircraft under various adverse conditions.

In this paper, we consider the control of the C-5 transport aircraft, which is similar to the Generic Transport Model [4]. We delineate the underlying nonlinear dynamic model and introduce various upsets, damages, and failures by representing these various adverse conditions in the form of uncertainties. We consider two different flight conditions that

This work was supported by the NASA IRAC project via NRA NNH07ZEA001N.

M. Matsutani (megumimemit.edu), T. Gibson, and A. M. Annaswamy are with the Department of Mechanical Engineering, Massachusetts Institute of Technology, Cambeidge, MA 02139, USA.

L. G. Crespo and J. Jang are with the National Institute of Aerospace, 100 Exploration Way, Hampton VA 23666 USA. focus on the longitudinal and lateral dynamics separately. An adaptive controller based on the linearized model of the C5 is designed, in conjunction with a baseline LQ-controller with integral action, and anti-windup components to accommodate magnitude saturation, similar to that proposed in [5], [6].

The resilience of the adaptive control architecture to uncertainty is evaluated for safety using the control verification methodology proposed in [7]. This methodology enables the determination of ranges of uncertainty for which a prescribed set of closed-loop requirements are satisfied.

\section{ThE GTM-Like AIRCRAFT}

In this section, we state the problem that will be considered in this paper. We begin with a description of the nonlinear dynamic model of C-5, a large transport aircraft whose aerodynamics data is available in [8]. We consider rigid body dynamics, aerodynamics, and the effect of the control inputs and derive the overall nonlinear flight model. We then discuss adverse conditions such as flight upsets, damages, and failures, and how they can be represented in the underlying model.

\section{A. A Nonlinear Dynamic Model}

A typical dynamic model of an aircraft consists of the equations of motion, aerodynamics, actuator dynamics, actuator saturation, and sensor dynamics. These can be compactly described as

$$
\dot{X}=F(X, \Lambda U)
$$

where

$$
\begin{gathered}
X=\left[\begin{array}{cccccccccccc}
V_{T} & \alpha & \beta & p & q & r & \phi & \theta & \psi & x & y & h
\end{array}\right]^{T} \\
U=\left[\begin{array}{lllllll}
e_{1} & e_{2} & a_{1} & a_{2} & r_{1} & r_{2}
\end{array}\right]^{T}
\end{gathered}
$$

which denotes the left and right elevator inputs, aileron inputs and lower and upper rudder inputs, respectively. Four throttle inputs are present, but are kept constant at their trim values, and are allowed to fail. $\Lambda$ describes the control effectiveness, and is equal to the identity under nominal conditions. For the purpose of control, Eq. (1) is linearized about a trim condition $\left(X_{0}, U_{0}\right)$ as LTI systems of the form

$$
\dot{x_{p}}=A_{p} x_{p}+B_{p} u+g\left(x_{p}, u\right)
$$

where

$$
\begin{gathered}
x_{p}=X-X_{0} \quad, \quad u=U-U_{0} \\
A_{p}=\left.\frac{\partial F(X, U)}{\partial X}\right|_{X_{0}, U_{0}}, \quad B_{p}=\left.\frac{\partial F(X, U)}{\partial U}\right|_{X_{0}, U_{0}}
\end{gathered}
$$


and $g\left(x_{p}, u\right)$ is higher order terms.

\section{B. Adverse Conditions}

We now describe the three categories of upsets, damages, and failures that we shall introduce in the above model. In all three cases, we will assume that the actuators are capable of magnitude saturation.

a) Flight upsets: The simplest adverse condition is considered in this section, which consists of a flight upset. This corresponds to changes in the initial condition of the state from trim. In particular, the response of the underlying closed-loop system to changes in $\alpha(0)$ needs to be evaluated. It is therefore easy to see that if a system is stable, guarantees for a bounded performance are automatically obtained. Whether the actual responses obtained, even though bounded, are actually within limits of what is an acceptable and safe performance remains to be demonstrated.

b) Flight damages leading to CG movement: A more serious condition that needs to be addressed is damage that occurs due to wing separation. This causes, among other things, the CG shift leading to an additional moment.

$$
\begin{aligned}
\Delta M & =-(L \cos \alpha+D \sin \alpha) \Delta x \\
\Delta L & =(L \cos \alpha+D \sin \alpha) \Delta y \\
\Delta N & =(D \cos \alpha-L \sin \alpha) \Delta y
\end{aligned}
$$

where $\Delta x$ and $\Delta y$ is the displacement from the $\mathrm{CG}$ to the reference location. The inertia tensor is adjusted accordingly [9]. These in turn change the aerodynamic forces and moments as well as the trim points $\left(X_{0}, U_{0}\right)$. Unlike the treatment in [10], we ignore the contribution of the centripetal component of the acceleration and only cases CG movements in the $x$ and $y$ directions.

c) Actuator Failures: We now consider a different adverse condition that can occur because of failures in control surfaces. Similar to [11], we represent these failures as $B_{p} \Lambda$ where $\Lambda$ is a matrix of dimension $6 \times 6$, whose elements are zero and one in the nominal case, and take values $\lambda_{i}$ in $(0,1)$, where $i$ refers to the specific actuator that has a loss of effectiveness due to a failure. For a general failure case when the effectiveness of each actuator is affected, $\Lambda$ is denoted as

$$
\Lambda=\operatorname{diag}\left[\begin{array}{llllll}
\lambda_{e 1} & \lambda_{e 2} & \lambda_{a 1} & \lambda_{a 2} & \lambda_{r 1} & \lambda_{r 2}
\end{array}\right]
$$

\section{Adaptive Control Architecture}

The overall adaptive control architecture includes a nominal controller that is designed so as to meet the Longitudinal FC and Lateral FC described in Section IV in the absence of any uncertainties. This is described in Section III-A. An adaptive controller is added to the nominal one to cope with various adverse conditions.

\section{A. Nominal Controller}

The nominal controller has three different components, an LQR controller with integral action, wash-out and low-pass filters to deal with measurement noise, and a hard-limiter to cope with possible saturation, whose limit is chosen to vary with the deflection of the elevator. Each of these components is described in more detail.

1) LQR Controller with Integral Action: We make an assumption that the pitch, yaw and roll dynamics are weakly decoupled. In addition, in order to closely follow the commanded angle of attach, an integral state $e_{\alpha}$ is added as

$$
e_{\alpha}=\int\left(\alpha-\alpha_{\mathrm{cmd}}\right) d t
$$

where $\delta_{\mathrm{cmd}}=\left[\begin{array}{lll}\delta_{\mathrm{e}, \mathrm{cmd}} & \delta_{\mathrm{a}, \mathrm{cmd}} & \delta_{\mathrm{r}, \mathrm{cmd}}\end{array}\right]^{T}$, and $\alpha_{\mathrm{cmd}}=10 \delta_{\mathrm{e}, \mathrm{cmd}}$. $\delta_{\mathrm{cmd}}$ is assumed to be generated by the pilot. The augmented plant dynamics is therefore described as

$$
\underbrace{\left[\begin{array}{c}
\dot{x}_{p} \\
\dot{e}_{\alpha}
\end{array}\right]}_{\dot{x}}=\underbrace{\left[\begin{array}{cc}
A_{p} & 0 \\
H & 0
\end{array}\right]}_{A} \underbrace{\left[\begin{array}{c}
x_{p} \\
e_{\alpha}
\end{array}\right]}_{x}+\underbrace{\left[\begin{array}{c}
B_{p} \\
0
\end{array}\right]}_{B_{1}} u+\underbrace{\left[\begin{array}{c}
0 \\
-I
\end{array}\right]}_{B_{2}} \alpha_{\mathrm{cmd}}
$$

Since the states of (9) are accessible, an LQR controller is designed as

$$
\begin{aligned}
& \delta_{e, n}=-\left[\begin{array}{lll}
K_{\delta_{e_{\alpha}}} & K_{\delta_{e_{q}}} & K_{\delta_{e_{\alpha}}}
\end{array}\right]\left[\begin{array}{c}
\alpha \\
q \\
e_{\alpha}
\end{array}\right] \\
& {\left[\begin{array}{l}
\delta_{a, n} \\
\delta_{r, n}
\end{array}\right]=-\left[\begin{array}{cc}
K_{\delta_{a_{p}}} & 0 \\
0 & K_{\delta_{r_{r}}}
\end{array}\right]\left[\begin{array}{l}
p \\
r
\end{array}\right]+\underbrace{\left[\begin{array}{c}
\delta_{a, c m d} \\
\delta_{r, c m d}
\end{array}\right]}_{K_{r} r}}
\end{aligned}
$$

where the control gains $K_{\delta .} \mathrm{s}$ are chosen so as to minimize the cost function

$$
J=\int\left(x^{T} R_{x x} x+u^{T} R_{u u} u\right) d t
$$

where $R_{x x}, R_{u u}$ are the weighting matrices. Eqs. (8)- (10) denote the linearized GTM-model dynamics together with the LQR controller with integral action as the nominal controller (see [9] for more details).

2) Washout Filters and Low-pass Filters: Since the actual GTM model includes washout filters $G_{w}(s)$ and low pass filters $G_{l}(s)$ in the system, these filters were included in our simulation model considered in this paper, and were of the form

$$
x_{w}=\left[G_{w}(s) G_{l}(s) I\right] x
$$

with

$$
G_{w}(s)=\frac{s}{s+c_{1}}, \quad G_{l}(s)=\frac{c_{2}}{s+c_{2}} .
$$

\section{B. Saturation}

In order to ensure that the control input does not exceed the prescribed limit for the three actuators, a rectangular saturation component $R_{s}\left(u_{i}\right)$ is included in the simulation model. This is defined as

$$
R_{s}\left(u_{i}\right)= \begin{cases}u_{i} & \text { if }\left\|u_{i}\right\| \leq u_{i, \max } \\ u_{i, \max } \operatorname{sign}\left(u_{i}\right) & \text { if }\left\|u_{i}\right\|>u_{i, \max }\end{cases}
$$

The bound on the integrated state $e_{\alpha}$ is obtained via an antiwindup like action as 


$$
R_{e}\left(e_{\alpha}, \delta_{e}(t)\right)= \begin{cases}e_{\alpha} & \text { if } \dot{e}_{\alpha} \geq 0 \text { or } e_{\alpha} \leq e_{\mathrm{b}}, \\ e_{\mathrm{b}} & \text { if } \dot{e}_{\alpha}<0 \text { and } e_{\alpha}>e_{\mathrm{b}}\end{cases}
$$

where the bound $e_{\mathrm{b}}$ is computed as

$$
\begin{aligned}
e_{\mathrm{b}} & =\max \left(0,\left(R_{S}\left(\delta_{e}\right)-\left(\delta_{e, \text { trim }}+\delta_{e, \text { stab }}\right)\right) / K_{\delta_{e_{\alpha}}}\right) \\
\delta_{e, \text { stab }} & =K_{\delta_{e_{\alpha}}} \alpha+K_{\delta_{e_{q}}} q_{w}
\end{aligned}
$$

Defining the saturation errors as

$$
\begin{aligned}
u_{\Delta} & =u-R_{s}(u) \\
e_{\alpha, \Delta} & =e_{\alpha}-R_{e}\left(e_{\alpha}, \delta_{e}(t)\right)
\end{aligned}
$$

the closed-loop system with the nominal controller, filters, and saturation can be expressed as follows.

$$
\begin{aligned}
\underbrace{\left[\begin{array}{c}
\dot{x}_{p} \\
\dot{e}_{\alpha}
\end{array}\right]}_{\dot{x}} & =\underbrace{\left[\begin{array}{cc}
A_{p}-B_{p} K_{x_{p}} & -B_{p} K_{\delta_{e_{\alpha}}} \\
H & 0
\end{array}\right]}_{A_{m}} \underbrace{\left[\begin{array}{c}
x_{p} \\
e_{\alpha}
\end{array}\right]}_{x}+\underbrace{\left[\begin{array}{c}
B_{p} \\
0
\end{array}\right]}_{B_{1}} K_{r} \delta_{c m d} \\
& +\underbrace{\left[\begin{array}{c}
0 \\
-I
\end{array}\right]}_{B_{2}} \alpha_{c m d}-\underbrace{\left[\begin{array}{c}
B_{p} \\
0
\end{array}\right]}_{R_{1}} u_{\Delta}-\underbrace{\left[\begin{array}{c}
-B_{p} K_{\delta_{e_{\alpha}}} \\
0
\end{array}\right]}_{R_{2}} e_{\alpha, \Delta}
\end{aligned}
$$

The boundedness of the closed-loop system can be established for all initial conditions inside a bounded set. This bound extends to the entire state-space if the open-loop plant is stable.

\section{Reference Model}

We choose a reference model as the nonlinear model (1) together with the nominal controller in (10) and filters (12) in the loop, without any uncertainties. Let $x_{m}$ be the states of the reference model. The resulting closed-loop system can be shown to have bounded solutions in a neighborhood of the trim condition. As compared to a linear reference model, the usage of a nonlinear one improves the system performance.

\section{Adaptive Controller}

We augment the controller in (10) with an adaptive component as follows:

$$
u=\delta_{n}=\left(K+\theta_{x}\right) x+\left(K_{r}+\theta_{r}\right) r+\hat{f}
$$

where $\theta_{x}, \theta_{r}$, and $\hat{f}$ are adjusted so as to minimize the error between $x$ and $x_{m}$. Defining the state error $e$ as

$$
e=x-x_{m}
$$

we choose the adaptive laws for adjusting the adaptive parameters in (19) as

$$
\begin{aligned}
\dot{\theta}_{x} & =-\Gamma_{1} B_{1}^{T} P e_{u} x^{T}-\sigma_{1} \theta_{x} \\
\dot{\theta}_{r} & =-\Gamma_{2} B_{1}^{T} P e_{u} r-\sigma_{2} \theta_{r} \\
\dot{\hat{f}} & =-\Gamma_{3} B_{1}^{T} P e_{u}-\sigma_{3} \theta_{f} \\
\dot{\hat{\lambda}} & =-\Gamma_{4} \operatorname{diag}(\Delta u) B_{1}^{T} P e_{u}-\sigma_{4} \hat{\lambda}
\end{aligned}
$$

where $A_{m}^{T} P+P A_{m}=-Q, Q>0$, and $\Gamma_{i}$ is diagonal and positive definite for $i=1, \ldots 4$. Also $e_{u}=e-e_{\Delta}$, where the auxiliary error $e_{\Delta}$ is defined as

$$
\dot{e}_{\Delta}=A_{m} e_{\Delta}-R_{1} \operatorname{diag}(\hat{\lambda}) u_{\Delta}
$$

$e_{\Delta}$ represents the error that occurs due to saturation, and by subtracting it out from $e$, we obtain a new error $e_{u}$ which represents the sum of the error due to uncertainties and the error due to $e_{\alpha, \Delta}$.

\section{E. Stability of the closed-loop system}

It should be noted that the stability and boundedness of the closed-loop system has been established in [5], [12], [6] where no anti-windup like saturation components are present. The stability of the adaptive system that is proposed here can also be guaranteed, the details of which will appear in [13].

\section{CONTROL Verification}

In this section we evaluate the improvements resulting from augmenting the baseline controller with an adaptive component. This is attained by determining the largest hyperrectangular set in the uncertain parameter space $p$ for which a set of closed-loop requirements are satisfied by all the set members. The section that follows presents a brief introduction to the mathematical framework required to perform this study. References [7] and [14] cover this material in detail.

\section{A. Mathematical Framework}

The parameters which specify the closed-loop system are grouped into two categories: uncertain parameters, which are denoted by the vector $p$, and the control design parameters, which are denoted by the vector $d$. While the plant model depends on $p$, the controller depends on $d$. The Nominal Parameter value, denoted as $\bar{p}$, is a deterministic estimate of the true value of $p$.

Stability and performance requirements for the closed-loop system will be prescribed by the set of inequality constraints, $g(p, d)<0$. Throughout this paper, it is assumed that vector inequalities hold component wise. For a fixed $d$, the larger the region in $p$-space where $g<0$, the more robust the controller.

The Failure Domain corresponding to the controller with parameters $d$ is given by ${ }^{1}$

$$
\begin{gathered}
\mathcal{F}^{j}(d)=\left\{p: g_{j}(p, d) \geq 0\right\}, \\
\mathcal{F}(d)=\bigcup_{j=1}^{\operatorname{dim}(\boldsymbol{g})} \mathcal{F}^{j}(d) .
\end{gathered}
$$

While Equation (23) describes the failure domain corresponding to the $j$ th requirement, Equation (24) describes the failure domain for all requirements. The Non-Failure Domain is the complement set of the failure domain and will be denoted ${ }^{2}$ as $C(\mathcal{F})$. The names "failure domain" and "non-failure domain" are used because in the failure domain

\footnotetext{
${ }^{1}$ Throughout this section, super-indices are used to denote a particular vector or set while numerical sub-indices refer to vector components, e.g., $p_{i}^{j}$ is the $i$ th component of the vector $p^{j}$.

${ }^{2}$ The complement set operator will be denoted as $C(\cdot)$.
} 
at least one constraint is violated while, in the non-failure domain, all constraints are satisfied.

Let $\Omega$ be a set in $p$-space, called the Reference Set, whose geometric center is the nominal parameter $\bar{p}$. The geometry of $\Omega$ will be prescribed according to the relative levels of uncertainty in $p$. One possible choice for the reference set is the hyper-rectangle

$$
\mathcal{R}(\bar{p}, n)=\{p: \bar{p}-n \leq p \leq \bar{p}+n\} .
$$

where $n>0$ is the semi-diagonal of the rectangle. In what follows we assume that $g(\bar{p}, d)<0$. The tasks of interest is to assign a measure of robustness to a controller based on measuring how much the reference set can be deformed before intersecting the failure domain. A homothet of $\Omega$ is given by the set $\{\bar{p}+\alpha(p-\bar{p}): p \in \Omega\}$, where $\bar{p}$ is the center of the rectangle and $\alpha>$, is the Similitude Ratio. While expansions of $\Omega$ are accomplished when $\alpha>1$, contractions result when $0 \leq \alpha<1$.

Intuitively, one imagines that a homothet of the reference set is being deformed until its boundary touches the failure domain. Any point where the deforming set touches the failure domain is a Critical Parameter Value (CPV). The $\mathrm{CPV}$, which will be denoted as $\tilde{p}$, might not be unique. The deformed set is called the Maximal Set (MS) and will be denoted as $\mathcal{M}$. The Critical Similitude Ratio, denoted as $\tilde{\alpha}$, is the similitude ratio of that deformation. While the critical similitude ratio is a non-dimensional number, the Parametric Safety Margin (PSM), denoted as $\rho$ and defined later, is its dimensional equivalent. Both the critical similitude ratio and the PSM quantify the size of the MS. Details on how to calculate the CPV $\tilde{p}$ and $\tilde{\alpha}$ are available in [7].

Once the CPV has been found, the MS is uniquely determined by

$$
\mathcal{M}(d)=\mathcal{R}(\bar{p}, \tilde{\alpha} n)
$$

The size of this set is proportional to the PSM which is defined as

$$
\rho=\tilde{\alpha}\|n\|
$$

Because the critical similitude ratio and the PSM measure the size of the MS, their values are proportional to the degree of robustness of the controller associated with $d$ to uncertainty in $p$. The critical similitude ratio is nondimensional, but depends on both the shape and the size of the reference set. The PSM has the same units as the uncertain parameters, and depends on the shape, but not the size, of the reference set. If the PSM is zero, the controller's robustness is practically nil since there are infinitely small perturbations of $\bar{p}$ leading to the violation of at least one of the requirements. If the PSM is positive, the requirements are satisfied for parameter points in the vicinity of the the nominal parameter point. The larger the PSM, the larger the hyperrectangular-shaped vicinity. Note that when $\operatorname{dim}\{p\}=1$, the PSM is $\rho=\left|\tilde{p}^{k}-\bar{p}\right|$ and the MS is $\mathcal{M}(d)=(\bar{p}-\rho, \bar{p}+\rho)$.

\section{B. Analysis Setup}

1) Uncertain Parameters: We will consider the following set of uncertain parameters

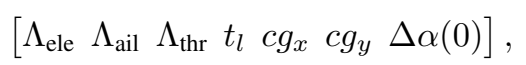

where the first 3 components are the control effectiveness of elevators, ailerons, and left engine throttle. In particular, $\Lambda . s$ are

$$
\begin{aligned}
\Lambda_{\text {ele }}=\left[\begin{array}{ll}
\lambda_{e 1} & \lambda_{e 2}
\end{array}\right], \Lambda_{\text {ail }} & =\left[\begin{array}{ll}
\lambda_{a 1} & \lambda_{a 2}
\end{array}\right], \Lambda_{\text {rud }}=\left[\begin{array}{lll}
\lambda_{r 1} & \lambda_{r 2}
\end{array}\right] \\
\Lambda_{\text {thr }} & =\left[\begin{array}{llll}
\lambda_{t 1} & \lambda_{t 2} & \lambda_{t 3} & \lambda_{t 4}
\end{array}\right]
\end{aligned}
$$

The term $t_{l}$ is the control surface lock-in-place duration. The terms $c g_{x}$ and $c g_{y}$ are components of the position vector on the $x y$-plain of the body frame of the CG location with respect to a reference point. The last component, which models a flight upset, is the initial condition in angle of attack. The nominal parameter values corresponding to this vector are $[1,1,1,0,0,0,0]$.

2) Closed-loop Requirements: The following stability and performance requirements will be considered

$$
\begin{gathered}
g_{0}=\max \left\{\left[u_{\text {trim }}-u_{\text {max }}, u_{\text {min }}-u_{\text {trim }}\right]\right\} \\
g_{1}=\max _{t}\left\{\frac{\left|a_{C G}\right|}{g}\right\}-2.5 \\
g_{2}=\left[\left(\alpha-\alpha_{\mathrm{cmd}}\right)^{2}+k_{\alpha} \dot{\alpha}^{2}+\left(\beta-\beta_{\mathrm{cmd}}\right)^{2}+k_{\beta} \dot{\beta}^{2}\right]_{t=t_{f}}-c_{1}, \\
g_{3}=\eta(p, d)-c_{2} \eta\left(\bar{p}, d_{\text {base }}\right), \\
\eta=w_{1}\left\|\alpha-\alpha_{\mathrm{cmd}}\right\|_{2}+w_{2}|| p-p_{\mathrm{cmd}}\left\|_{2}+w_{3}\right\| r-r_{\mathrm{cmd}} \|_{2} .
\end{gathered}
$$

The first requirement, $g_{0}<0$, is used to determine if the vehicle has enough control authority to trim, i.e. if it satisfies $u_{\min }<u_{\text {trim }}<u_{\max }$. Note that this requirement is independent on $d$ and may indicate instability. $g_{1}<0$, where $a_{C G}$ is the acceleration at the $\mathrm{CG}$, is a structural integrity requirement enforced by preventing the loading factor from exceeding 2.5. The requirement $g_{2}<0$, where $0<c_{1} \ll 1$, $k_{\alpha}>0$ and $k_{\beta}>0$, enforces stability and satisfactory steady state performance. The last requirement, $g_{3}<0$, for $c_{2}>1, w_{1}>0, w_{2}>0$ and $w_{3}>0$, is used to measure satisfactory transient performance. This requirement prevents the cumulative error from exceeding a prescribed upper limit. Such a limit is assumed to be larger than the cumulative error corresponding to the baseline controller $d_{\text {base }}$ for the nonuncertainty case.

Numerical studies showed that the trim-ability requirement $g_{0}<0$ is satisfied as long as the other requirements are satisfied, i.e., $\mathcal{F}^{0} \subset \bigcup\left\{\mathcal{F}^{1}, \mathcal{F}^{2}, \mathcal{F}^{3}\right\}$. Therefore, the studies presented subsequently will only consider $g_{1}, g_{2}$, and $g_{3}$.

In practice, control requirements are prescribed in advance before the control design process even starts. When such requirements are only described qualitatively several 
TABLE I

CASES ANALYZED. 1-DIMENSION SEARCHES ARE CONDUCTED FOR THE SHOWN SETS OF A FAILURE/UNCERTAINTY AND AN APPROPRIATE FC.

\begin{tabular}{|c|c|c|}
\hline Case & Failure/Uncertainty & \\
\hline Case A & Damage 1: CG change along $y$-axis & {$\left[c g_{y} f_{\text {lat }}\right]$} \\
\hline Case B & Damage 2: CG change along $\mathrm{x}$-axis & {$\left[\begin{array}{cl}c g_{x} & f_{\text {lon }}\end{array}\right]$} \\
\hline Case $\mathrm{C}$ & Flight upset with angle of attack & {$\left[\Delta \alpha(0) f_{\text {lon }}\right]$} \\
\hline Case D & Failure 1: Symmetric Aileron failures & {$\left[\Lambda_{\text {ail }} f_{\text {lat }}\right]$} \\
\hline Case $\mathrm{E}$ & Failure 2: Symmetric Elevator failures & {$\left[\Lambda_{\text {ele }} f_{\text {lon }}\right]$} \\
\hline Case F & Failure 3: Asymmetric Aileron failure & {$\left[\begin{array}{ll}\lambda_{\mathrm{a} 1} & f_{\text {lat }}\end{array}\right]$} \\
\hline Case $\mathrm{G}$ & Failure 4: Asymmetric Throttle failure & {$\left[\begin{array}{ll}\lambda_{\mathrm{t} 1} & f_{\text {lon }}\end{array}\right]$} \\
\hline Case $\mathrm{H}$ & Failure 5: Lock-in-place failure & {$\left[t_{l} f_{\text {lon }}\right]$} \\
\hline
\end{tabular}

$g$ implementations are possible. This creates the additional challenge of constructing functional forms that capture well the intent of the requirement while having a minimal amount of conservatism. This paper does not tackle such a challenge and assumes that the $g$ above is given.

\section{Flight Conditions (FC)}

The closed-loop response depends on $p$ and $d$ as well as in the intended flight maneuver $f$. This implies that $g(p, d, f)$. Two flight conditions, namely $f_{\text {lon }}$ and $f_{\text {lat }}$, will be considered in the analyses that follow. In the former one, which mostly affects the longitudinal dynamics, the vehicle starts from level flight and reaches a fixed angle of attack after a short transient. In the second one, which affects both the longitudinal and lateral dynamics, the vehicle also starts from level flight and turns.

\section{RESUlTs}

Aerodynamic data and configuration for C-5 can be found in [8]. While the trim values of the velocity, angle of attack, and height are $614 \mathrm{ft} / \mathrm{s}, 2.2 \mathrm{deg}$ and $20000 \mathrm{ft}$ respectively all other numerical values used are in [9].

In this section, we evaluate the adaptive controller defined in eqs. (19)-(22) in the presence of flight upsets, damages that cause CG movement, and actuator failures.

In Case A we consider the movement of the $\mathrm{CG}$ in the $y$-direction for the lateral flight condition. Recall that a positive CG movement denotes movement to the right. The dependency of $g$ on the CG location for both controllers is illustrated in Figure 1. The dashed lines and the solid lines represent results from the baseline and adaptive controllers, respectively. A comparison of these curves shows that the non-failure region of the adaptive controller is larger by virtue of the steady state performance and the transient performance requirements. The baseline controller has a PSM of 0.0029 while the adaptive one attains a PSM of 0.0069 . The curves are asymmetric with respect to the nominal parameter value, since the flight condition is itself asymmetric.

In Case B we consider the movement of the CG in the $x$-direction for the longitudinal flight condition. Recall that a positive value of the CG movement denotes movement forwards. The dependency of $g$ on the CG location for both

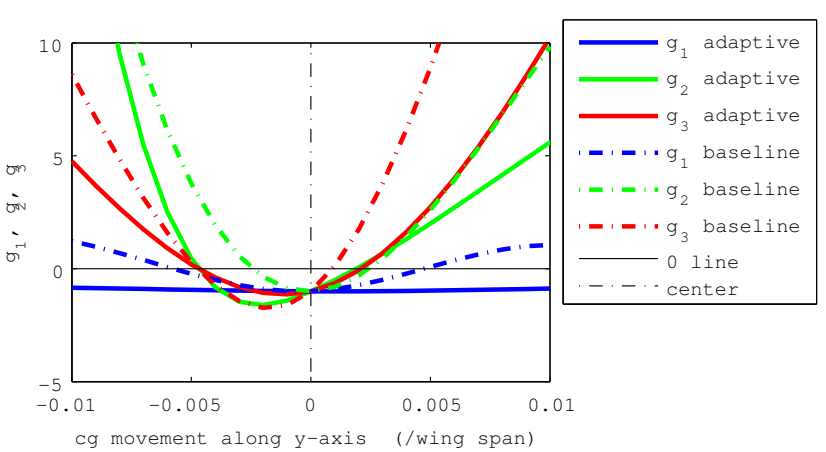

Fig. 1. Case C: $g\left(c g_{y}\right)$ for the lateral FC.

controllers is studied with the control verification metric introduced in Section IV, and it is shown that the system loses stability when the CG moves backward, while the tracking performance degrades the faster when the CG moves forward. The baseline controller has a PSM of 0.175 while the adaptive one attains a PSM of 0.197.

In Case $\mathrm{C}$ we consider a flight upset in the angle of attack, $\Delta \alpha(0)$ about $\alpha_{\text {trim }}=2.20(\mathrm{deg})$ for the longitudinal flight condition. The dependency of $g$ on $\Delta \alpha(0)$ for both controllers is again studied. A comparison of the curves shows that the non-failure region of the adaptive controller is larger by virtue of the loading factor requirement and the tracking performance requirement.

In Case $\mathrm{D}$, we consider a symmetric failure in both ailerons, where $\lambda_{a i l}=\lambda_{a_{1}}=\lambda_{a_{2}}$, for the lateral FC. While the resulted PSM for the baseline is $6.6 \%$, the PSM for the adaptive is $10 \%$. In both cases, the tracking performance is the critical requirement.

In Case $\mathrm{E}$ we consider a symmetric failure in both elevators, where $\lambda_{\text {ele }}=\lambda_{e_{1}}=\lambda_{e_{2}}$, for the longitudinal FC. While the resulted PSM for the baseline is $33 \%$, the PSM for the adaptive is $42 \%$. In both cases, the tracking performance is the critical requirement. As before, the adaptive controller has better robustness characteristics.

Unlike Case C, Case F considers an asymmetric aileron failure for the lateral FC where $\lambda_{a_{1}}$ is uncertain and $\lambda_{a_{2}}=$ 1. While the resulted PSM for the baseline is $14 \%$, the PSM for the adaptive is $20 \%$. Consistently, the tracking performance requirement remains being the critical, while the PSM corresponding to the stability requirement drops.

In Case $\mathrm{G}$ we consider a failure in the left outboard engine $\lambda_{t 1}$ for the longitudinal FC. While the resulted PSM for the baseline is $1.7 \%$, the PSM for the adaptive is $2.9 \%$. As before, the tracking performance is the critical requirement. Note that the margins obtained in this case are considerably smaller than those found in the others. The non-failure domains are small since the throttle inputs are not controlled but rather fixed at their trim values. Similar results were observed with the Lateral FC.

A lock-in-place failure in the left elevator is considered in Case H. This is simulated by keeping this control input at a constant value for a period of $t_{l}$ seconds. The larger the 
TABLE II

SUMMARY FOR THE RESULTS OF ALL CASES

\begin{tabular}{|l||c|c|}
\hline Case & $\left(\frac{\rho_{\text {adap }}}{\rho_{\text {base }}}-1\right) \times 100 \%$ & Critical Requirement \\
\hline A & $+133 \%$ & $g_{2}, g_{3}$ \\
\hline B & $+11.4 \%$ & $g_{2}, g_{3}$ \\
\hline C & $+4.01 \%$ & $g_{1}, g_{3}$ \\
\hline D & $+63.6 \%$ & $g_{3}$ \\
\hline E & $+27.3 \%$ & $g_{3}$ \\
\hline F & $+46.7 \%$ & $g_{3}$ \\
\hline G & $+70.6 \%$ & $g_{3}$ \\
\hline H & $+88.9 \%$ & $g_{2}, g_{3}$ \\
\hline
\end{tabular}

$t_{l}$ the more severe the failure. In Case $\mathrm{H}$, there are present substantial differences in the functional dependencies. It is seen that the PSM for the baseline is 1.1 while the PSM for the adaptive is 2.1. Note also that while the tracking performance is critical for the baseline, the stability requirement is critical for the adaptive one.

Table II summarizes the results above by presenting the relative change in PSM attained by the adaptive controller and the critical requirement. In all cases, the adaptive controller attains better robustness by a sizable margin.

\section{A. Multi-dimensional Case}

In all the studies above uncertainty in a single parameter has been considered. In this setting, the effect of the dependencies among parameters are not captured. The same analysis can be conducted for a multi-dimensional vector $p$. In such a case, multiple failures and uncertainties occur simultaneously and the correlation among them may play a significant role. Studies of this type will be presented elsewhere. However, Figure 2 presents a time simulation of the controlled response for a multi-dimensional parameter realization when 2 pitch doublets are commanded. Therein, we assume losses in control effectiveness of $30 \%$ for the elevators, $10 \%$ for the ailerons, and $10 \%$ for the rudders. Besides, the CG has been moved to the left by $0.004 / c$, and a flight upset in the angle of attack of 0.2 degrees is assumed. It is apparent that the adaptive controller achieves good tracking performance while the nominal controller can not recover and becomes unstable.

\section{SUMmary}

This paper presents an adaptive control architecture for the safe flight of a transport aircraft under adverse operating conditions and uncertainties. This architecture combines a nominal controller based on an LQR with integral action, and an adaptive controller that accommodates for actuator saturation and disturbances. The resilience of both controllers to uncertainty is studied using a control verification methodology, where flight upsets, CG movements, and actuator failures are considered. The results of this study show that the adaptive controller enlarges the region of satisfactory performance by a sizable margin.
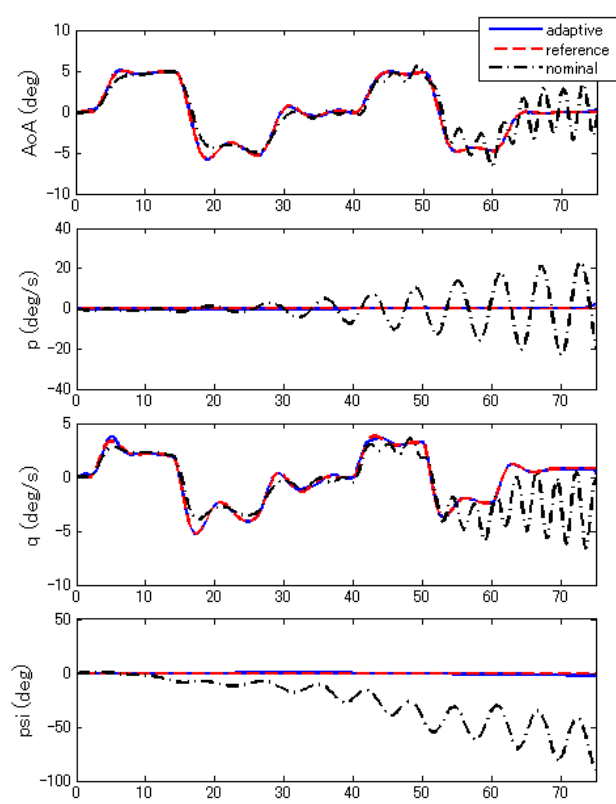

Fig. 2. Time response result with combination of multiple failures and uncertainties

\section{REFERENCES}

[1] Narendra, K. S.; and Annaswamy, A. M.: Stable Adaptive Systems. Prentice-Hall, Englewood Cliffs, NJ, 1989.

[2] Ioannou, P. A.; and Sun, J.: Robust Adaptive Control. Prentice-Hall, Upper Saddle River, NJ, 1996.

[3] Tao, G; and Kokotovic, PV: Adaptive control of systems with actuator and sensor nonlinearities. John Wiley and Sons, Inc., New York, NY, 1996.

[4] Bailey, R. M.; Hostetler, R. W.; Barnes, K. N.; and Belcastro, C. M.: Experimental Validation: Subscal Aircraft Ground Facilities and Integrated Test Capability. AIAA Guidance, Navigation, and Control Conference, 2005, AIAA-2005-6433.

[5] Karason, S. P.; and Annaswamy, A. M.: Adaptive Control in the Presence of Input Constraints. IEEE Transaction on Automatic Control, vol. 46, no. 11, November 1994, pp. 2325-2330.

[6] Jang, J.; Annaswamy, A. M.; and Lavretsky, E.: Adaptive Flight Control in the Presence of Multi-input Magnitude Saturation. American Control Conference, 2008.

[7] Crespo, L. G.; Giesy, D. P.; and Kenny, S. P.: Robust Control Analysis from a System Requirements Perspective. NASA TP 2009-000001, May 2009.

[8] Heffley, R. K.; and Jewell, W. F.: Aircraft Handling Qualities Data. Contractor's Report CR-2144, NASA, Systems Technology, Inc. Hawthorne, CA 90250, December 1972. Available at ntrs.nasa.gov.

[9] Matsutani, M.; Jang, J.; Gibson, T.; and Crespo, L. G.: On the Quantifcation of the Improvements Resulting from Adaptive Control: a Proof of Concept. Tech. rep., NASA-CR, 2009.

[10] Bacon, J. B.; and Gregory, M. I.: General Equations of Motion for a Damaged Asymmetric Aircraft. AIAA Atmospheric Flight Mechanics Conference and Exhibit, Hilton Head, South Carolina, August 2007, AIAA-2007-6306.

[11] Jang, J; Annaswamy, A. M.; and Lavretsky, E.: Adaptive Flight Control in the Presence of Multiple Actuator Anomalies. American Control Conference, New York, NY, 2007, pp. 3300-3305.

[12] Schwager, M.; and Annaswamy, A. M.: Adaptive Control of MultiInput Systems with Magnitude Saturation Constraints. Proc. Conference on Decision and Control, Seville, Spain, December 2005, pp. 783-788.

[13] Matsutani, M.; Crespo, L. G.; and Annaswamy, A. M.: An Adaptive Control Technology for Safe High-Performance Aircraft. Master's thesis, Massachusetts Institute of Technology, 2009 (in preparation).

[14] Crespo, L. G.; Giesy, D. P.; and Kenny, S. P.: Robust Analysis and Robust Design of Uncertain Systems. AIAA Journal, vol. 46, no. 2, 2008. 